\title{
Let-7d functions as novel regulator of epithelial-mesenchymal transition and chemoresistant property in oral cancer
}

\author{
CHARN-JUNG CHANG ${ }^{1}$, CHUAN-CHIH HSU $^{2}$, CHIN-HONG CHANG $^{2}$, LO-LIN TSAI ${ }^{3,4}$, YU-CHAO CHANG $^{3,4}$, \\ SHAO-WEI LU ${ }^{1}$, CUUAN-HANG YU ${ }^{3,4}$, HSU-SHAN HUANG ${ }^{1}$, JHI-JOUNG WANG ${ }^{2}$, CHUNG-HUNG TSAI $^{5}$, \\ MING-YUNG CHOU ${ }^{3,4}$, CHENG-CHIA YU $\mathrm{U}^{3,4,6}$ and FANG-WEI HU ${ }^{4,6}$
}

\begin{abstract}
${ }^{1}$ Department of Pharmacy Practice, Tri-Service General Hospital and National Defense Medical Center, Taipei; ${ }^{2}$ Department of Surgery, Chi-Mei Medical Center and Chia Nan University of Pharmacy and Science, Tainan; ${ }^{3}$ Institute of Oral Biology and Biomaterial Science, ${ }^{4}$ School of Dentistry, College of Oral Medicine and ${ }^{5}$ Institute of Medicine, Chung Shan Medical University, Taichung; ${ }^{6}$ Department of Dentistry, Chung Shan Medical University Hospital, Taichung, Taiwan, R.O.C.
\end{abstract}

Received April 20,2011; Accepted May 30, 2011

DOI: 10.3892/or.2011.1360

\begin{abstract}
Oral squamous cell carcinoma (OSCC) is a prevalent cancer worldwide. Let-7 family has been shown to function as a tumor suppressor through regulating multiple oncogenic signaling. Recent study reported that combined underexpression of miR-205 and let-7d showed negative correlation with the survival prognosis of head and neck cancer patients. However, the let-7d-involved mechanism in regulating OSCC is still unclear. In this study, we first demonstrated that let-7d expression was significantly decreased while Twist and Snail expression was increased in OSCC cancer cell lines and primary cultures as compared to normal human oral keratinocyte cells. To further investigate the role of let-7d in OSCC, we applied the SPONGE method to knock down let-7d in OECM-1 and two primary OSCC cell types. The results showed that knockdown of let-7d promote epithelialmesenchymal transition (EMT) traits and migratory/invasive capabilities in OSCC cells. Furthermore, down-expression of let-7d significantly activated Twist and Snail expressions and chemo-resistant abilities of OSCC cells. Notably, overexpression of let-7d effectively reversed the EMT phenotype, blocked migratory/invasive abilities, and further increased the chemosensitivity in oral cancer tumor initiating $\mathrm{ALDH}^{+}$cells. In sum, these results show that let-7d negatively modulates EMT expression and also plays a role in regulating chemo-resistant ability in oral cancer.
\end{abstract}

Correspondence to: Dr Fang-Wei Hu or Dr Cheng-Chia Yu, School of Dentistry, College of Oral Medicine, Chung Shan Medical University and Department of Dentistry, Chung Shan Medical University Hospital, no. 110, Sec. 1, Jianguo N. Rd., Taichung 40201, Taiwan, R.O.C.

E-mail: fang0989909009@gmail.com

E-mail: ccyu@csmu.edu.tw

Key words: let-7d, epithelial-mesenchymal transition, Twist, Snail, and chemo resistance

\section{Introduction}

Head and neck squamous cell carcinoma, including oral squamous cell carcinoma (OSCC), is the sixth most prevalent malignancy worldwide and accounts for approximately $8-10 \%$ of all cancers in Southeast Asia $(1,2)$. The prognosis of OSCC remains dismal as more than $50 \%$ of the patients die of this disease or complications within 5 years under current therapies (2). To increase patient survival rate, investigations elucidating the mechanisms of tumorigenesis in OSCC are urgently needed (2). Some studies have suggested that subsets of cancer stem cells (CSC) or tumor initiating cells (TICs) are responsible for tumor progression as well as recurrence after conventional chemotherapy (3). However, there is lack of suitable markers for isolating the crucial subset of tumor cells that is capable of reforming new tumors in vivo and accounts for tumor relapse in OSCC, according to CSC hypothesis of tumorigenesis.

MicroRNAs (miRNAs), highly conserved small RNA molecules that regulate gene expression, can act as cancer signatures, oncogenes or tumor suppressors (4). The ubiquitously expressed let-7/miR-98 family was one of the first mammalian miRNAs to be identified (5-8). Let-7 family members have been described as being down-regulated during cancer progression in various human cancers including lung, gastric, ovarian, colon cancer, leiomyoma and melanoma (5-13). Let-7d, a member of the let-7 family of miRNAs, has also been shown to act as tumor suppressor, most likely through targeting RAS (14) or high mobility group A2 (15). Let-7d regulates senescence of human cord blood-derived multipotent stem cells by regulating HMGA2 and p16 (16). The region upstream of let-7d promoter is modulated by transforming growth factor (TGF)- $\beta$ transcription factor SMAD3 (15). The expression of let-7d is significantly down-regulated in chemo-resistant pancreatic cancer (17). Dysregulation of let-7d also indicated its possible involvement in the development and progression of ovarian cancer (9), glioblastoma (18), lung cancer (15), colon cancer (19) and leukemia (20). Scapoli et al found that combined underexpression of miR-205 and let-7d 
was associated with poor survival of head and neck squamous cell carcinomas (21). However, the relationship between let-7d and OSCC and OSCC-derived tumor initiating cells is still not clarified.

In this study, we showed that expression of let-7d in regional metastatic lymph nodes of OSCC patients was also significantly decreased while Twist and Snail expression was increased as compared to the expression levels in the primary tumors. Using the SPONGE strategy, we knocked down the expression of let-7d, and the results of in vitro experiments showed that the epithelial-mesenchymal transition (EMT), migratory/invasive, and chemoresistant properties were significantly increased in primary let-7d knockdown OSCC cells. These results suggest that the down-expression of let-7d plays a crucial role in regulating EMT and sensitivity to chemotherapy in OSCC.

\section{Materials and methods}

OSCC cell line and primary cell cultures. OECM1 was obtained from the American Type Culture Collection (ATCC) and cultured as described previously (22). All samples were obtained with informed consent. The OSCC patient characteristics were described previously (22). The procedures and methods were as described previously (22).

miRNA isolation and miRNA quantitative real-time reversetranscriptase (RT)-PCR. A mirVana Paris kit (Ambion) was used to isolate miRNA from the total-RNA according to the manufacturer's instructions. For miRNAs detection, qRT-PCR was performed by using TaqMan miRNA assays (Applied Biosystems) with specific primer sets. All reagents and protocols were from Applied Biosystems and detection was performed by using a 7900HT fast real-time PCR system using RNU6B as internal controls. miRNA specific qRT-PCR was done in triplicate and repeated three times on the ABI PRISM 7700 Sequence Detector system (Applied Biosystems) (22).

SYBR real-time reverse transcription-polymerase chain reaction. Total-RNA of cells was purified using TRIzol reagent (Invitrogen Life Technologies, Carlsbad, CA) according to the manufacturer's protocol. Briefly, the total-RNA $(1 \mu \mathrm{g})$ of each sample was reversely transcribed by SuperScript II RT (Invitrogen). Then, the amplification was carried out in a total volume of $20 \mu \mathrm{l}$ containing $0.5 \mu \mathrm{M}$ of each primer, $4 \mathrm{mM} \mathrm{MgCl}$, $2 \mu 1$ LightCycler $^{\mathrm{TM}}$-FastStart DNA Master SYBR-Green I (Roche Molecular Systems, Alameda, CA) and $2 \mu \mathrm{l}$ of 1:10 diluted cDNA. The GAPDH housekeeping gene was amplified as a reference standard. GAPDH primers were designed: GAPDH (forward), GGGCCAAAAGGGTCATCATC (nt 414-434, GenBank accession no. BC059110.1); GAPDH (reverse), ATGACCTTGCCCAC AGCCTT (nt 713-733). PCR reactions were prepared in duplicate and heated to $95^{\circ} \mathrm{C}$ for $10 \mathrm{~min}$ followed by 40 cycles of denaturation at $95^{\circ} \mathrm{C}$ for $10 \mathrm{sec}$, annealing at $55^{\circ} \mathrm{C}$ for $5 \mathrm{sec}$, and extension at $72^{\circ} \mathrm{C}$ for $20 \mathrm{sec}$. All PCR reactions were performed in triplicate. Standard curves (cycle threshold values versus template concentration) were prepared for each target gene and for the endogenous reference $(G A P D H)$ in each sample. To confirm the specificity of the PCR reaction, $\mathrm{PCR}$ products were electrophoresed on a $1.2 \%$ agarose gel (23).
Stable overexpression of let-7d by lentiviral vector. Let-7d expressing vectors were constructed according to Invitrogen. Briefly, oligos containing mature and artificial let-7d stem loop sequences were synthesised and annealed. Annealed oligos were ligated by T4 DNA ligase with pcDNA 6.2-GW/ EmGFP-miR expression vector provided by Invitrogen. Lentiviral production was further performed by transfection of $293 \mathrm{~T}$ cells at $5 \times 10^{6}$ cells $/ 10 \mathrm{~cm}$ plate using Lipofectamine 2000 (LF2000, Invitrogen). Supernatants were collected $48 \mathrm{~h}$ after transfection and then were filtered; the viral titers were then determined by FACS at $48 \mathrm{~h}$ post-transduction. Subconfluent cells were infected with lentivirus at a multiplicity of infection of 5 in the presence of $8 \mu \mathrm{g} / \mathrm{ml}$ polybrene (Sigma-Aldrich). The green fluorescence protein (GFP), which was co-expressed in lentiviral-infected cells, served as a selection marker to indicate the successfully infected oral cancer.

Plasmid construction and establishment of stable cell lines. Let-7d SPONGE, scramble and anti-sense miRNAs were constructed using a pcDNA 6.2-GW/EmGFP-miR plasmid (Invitrogen). MicroRNA SPONGE sequence design was as described previsouly (24). Further multiple copy amplifications were done with recovery of $B a m \mathrm{H} 1$ and XhoI digested fragments and subcloned into pcDNA 6.2-GW/EmGFP-miR plasmid. All constructed expression vectors were sequenced and verified. Transfected glioma cells were cultured in the presence of blasticidin-containing culture medium.

Western blot analysis assay. The extraction of proteins from cells and immunoblot analysis were performed as described. Fifteen microliters of sample was boiled at $95^{\circ} \mathrm{C}$ for $5 \mathrm{~min}$ and separated on a $10 \%$ SDS-PAGE. The proteins were transferred to Hybond-ECL nitrocellulose paper or PVDF membrane (Amersham, Arlington Heights, IL) by a wet-transfer system. Primary and secondary antibodies were added as indicated. The reactive protein bands were detected by the ECL detection system (Amersham) (25).

In vitro cell invasion analysis and soft agar assay. The 24-well plate Transwell ${ }^{\circledR}$ system with a polycarbonate filter membrane was used ( $8 \mu \mathrm{m}$ pore size; Corning, UK). Cell suspensions were seeded in the upper compartment of the Transwell chamber at a density of $1 \times 10^{5}$ cells in $100 \mu 1$ of serum-free medium. The opposite surface of the filter membrane facing the lower chamber was stained with Hoechst 33342 for $3 \mathrm{~min}$, and migrating cells were visualized under an inverted microscope. For the soft agar assay, the bottom of each well $(35 \mathrm{~mm})$ of a six-well culture dish was coated with $2 \mathrm{ml}$ of an agar mixture [DMEM, $10 \%$ (v/v) FCS, 0.6\% (w/v) agar]. After the bottom layer solidified, $2 \mathrm{ml}$ of a top agar-medium mixture [DMEM, $10 \%$ (v/v) FCS, $0.3 \%$ (w/v) agar] containing $2 \times 10^{4}$ cells was added and incubated at $37^{\circ} \mathrm{C}$ for 4 weeks. The plates were stained with $0.5 \mathrm{ml} 0.005 \%$ crystal violet, and the number of colonies was counted using a dissecting microscope (26).

Statistical analysis. The Statistical Package of Social Sciences software (SPSS, Inc., Chicago, IL) was used for statistical analysis. An independent Student's t-test was used to compare the means of two independent samples. The statistical significance level was set at $\mathrm{P}<0.05$. 
A

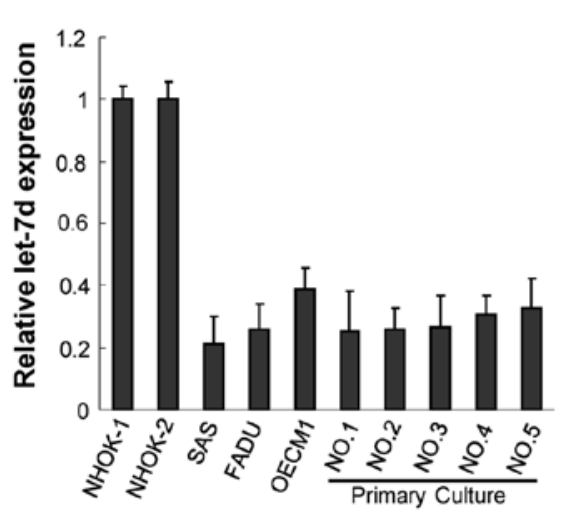

B

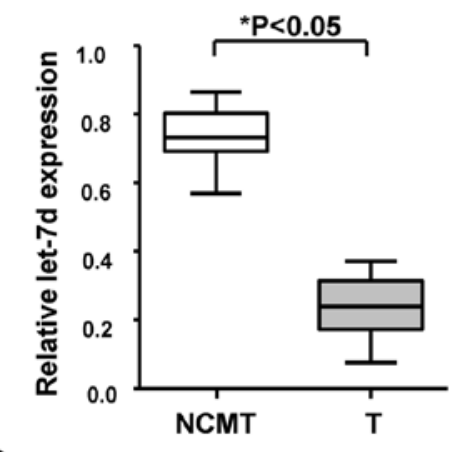

C

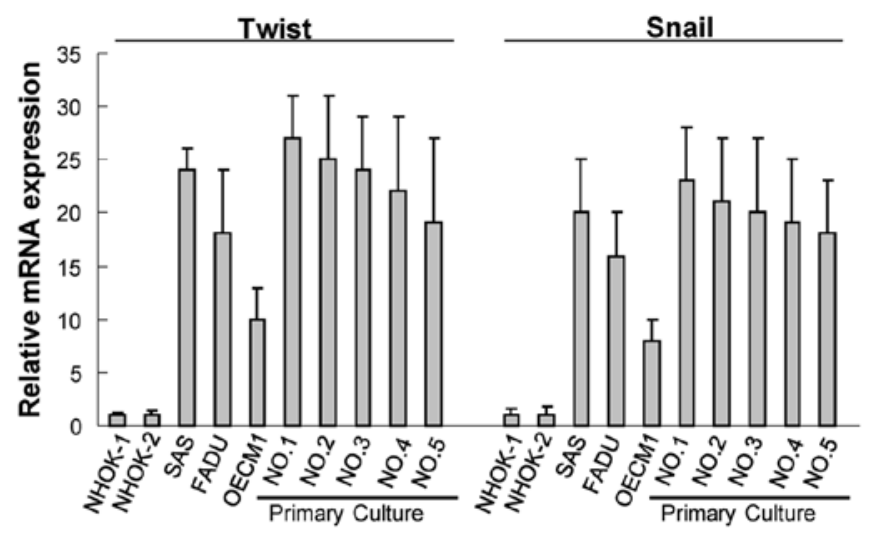

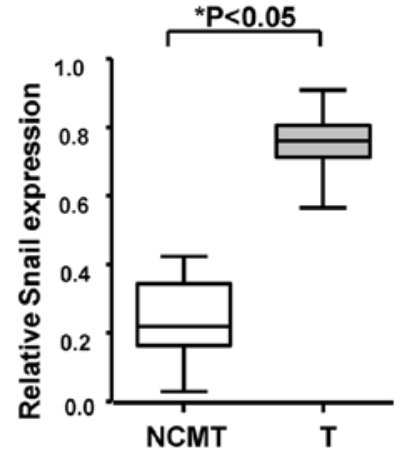

${ }^{\star} \mathrm{P}<0.05$

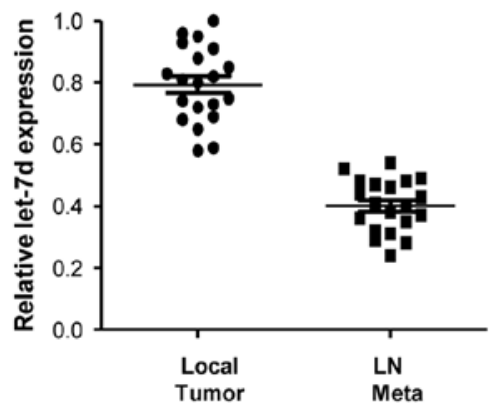

${ }^{\star} \mathrm{P}<0.05$

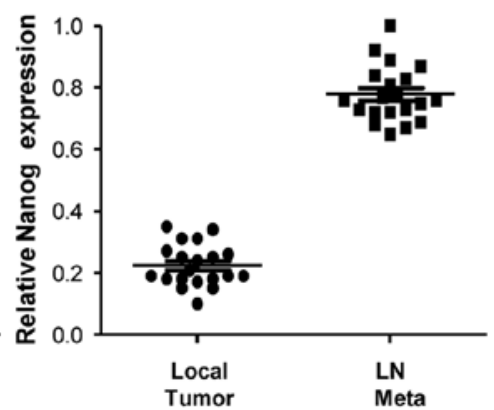

${ }^{*} \mathrm{P}<0.05$

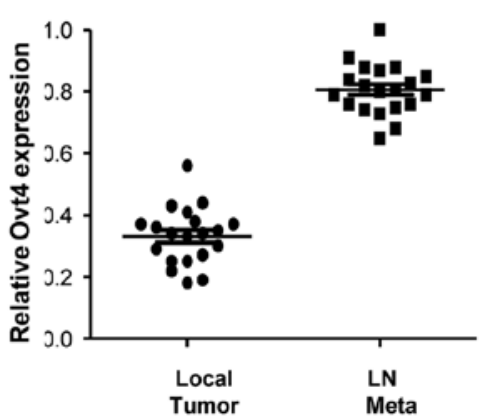

Figure 1. Determination of let-7d, Twist and Snail expression in representative OSCC tissue. (A) Let-7d and Twist and Snail expression in NHOK cells, oral cancer cell lines, and five primary OSCC cells. (B) A statistically significant difference of miRNA (let-7d) and mRNA expression (Twist and Snail) was also noted between OSCC (T) and corresponding noncancerous matched tissues (NCMT). (C) Expression level of let-7d microRNA (left panel), Twist (middle panel) and Snail (right panel) in local and lymph node metastatic head and neck cancer patients ("P<0.05).

\section{Results}

Let-7d negatively correlates with Twist and Snail in clinical OSCC patients. The correlation of miRNAs, stemness signature, and EMT process has recently gained huge interest in the field of cancer research, as it also contributes to tumor metastasis and recurrence (27). Initially, to investigate the negative relationship between the expression levels of let-7d and EMT genes in OSCC, the expression level of let-7d and EMT transcription markers (Twist and Snail) in OSCC cell lines, OSCC primary cultivated cells, and OSCC tissue specimens were determined. Compared with normal human oral keratinocyte (NHOK) cells, qRT-PCR demonstrated the lowered let-7d and elevated Twist and Snail expressions in OSCC cell lines and five primary OSCC cell types (Fig. 1A). Compared with the normal counterparts, further analysis showed a reduction of let-7d expression in OSCC tissue specimens derived from OSCC patients (Fig. 1B, left panel). Inversely, Twist and Snail expression is higher in OSCC tissue compared to that in normal tissue (Fig. 1B, middle and right panel). Finally, we demonstrated that let-7d expression in the regional metastatic lymph node of OSCC tissues was significantly decreased, whereas Twist and Snail expression was increased as compared to primary tumors (Fig. 1C). Collectively, these data indicate that let-7d expression is inversely correlated with EMT transcription factor (Twist and Snail) expression during OSCC tumorigenesis. 
A

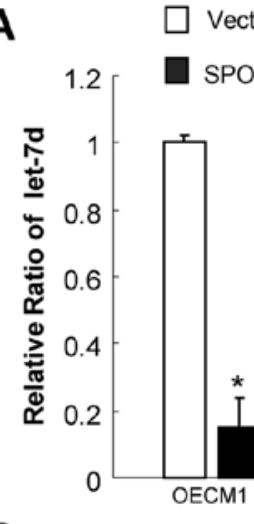

C

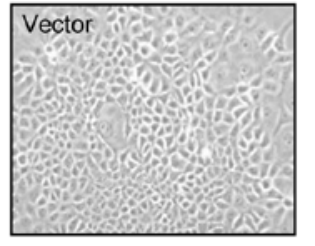

SPONGE-Let7d

Vector

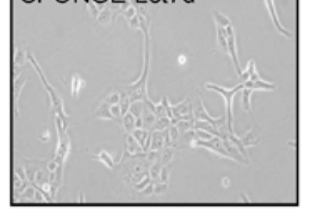

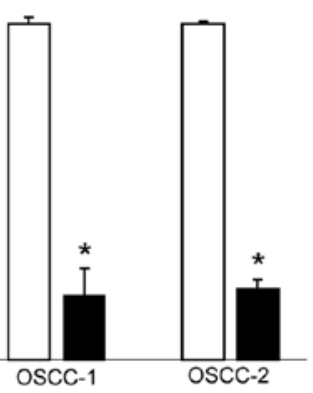

D

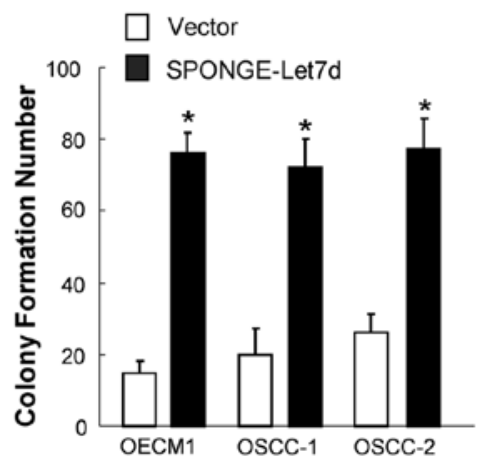

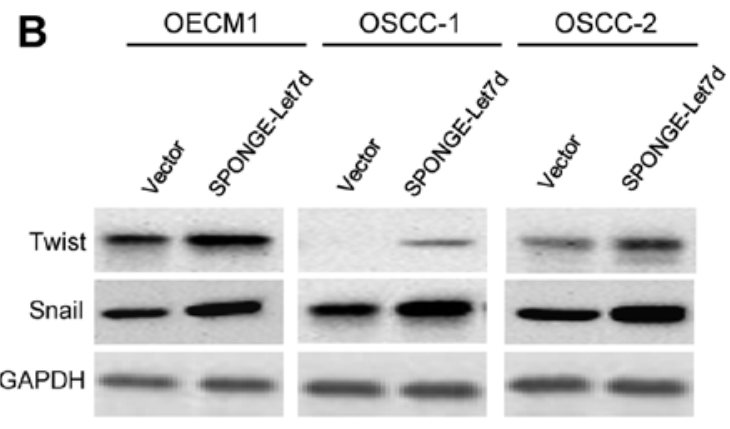

$\mathbf{E}$

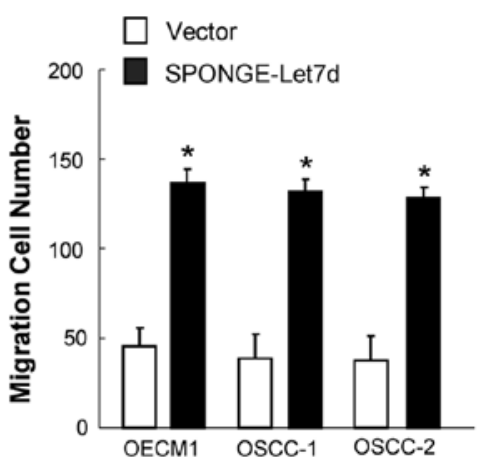

Figure 2. Down-regulation of let-7d restores EMT and invasive property in OSCC. (A) OECM1 and primary OSCC cells from two patients were transfected with either SPONGE let-7d or empty vector, and subjected to real-time PCR. (B) Expression of EMT transcription factor markers (Twist and Snail) of control and let-7d knockdown OSCC cells was assessed by Western blotting. (C) Morphology of OSCC cells transfected with control or let-7d SPONGE. Control OSCC cells and let-7d knockdown OSCC cells were subjected to (D) colony formation assay (left panel) and (E) migration assay (right panel).

A $\square$ vector

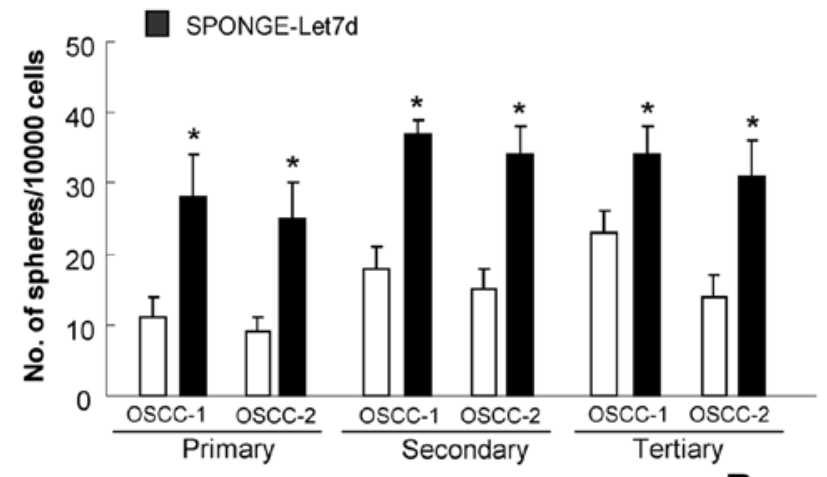

C

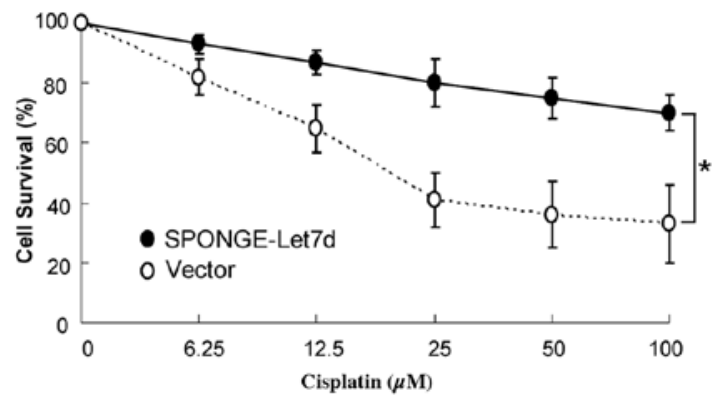

B

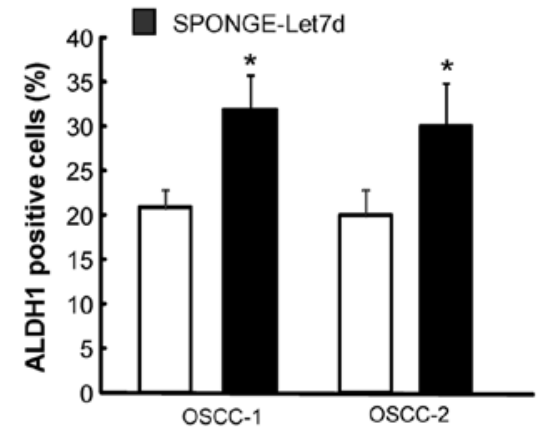

Figure 3. Sphere-forming and chemo-resistant property was enhanced in OSCC cells with let-7d SPONGE treatment. (A) Control OSCC cells and let-7d knockdown OSCC cells were subjected to sphere formation assays after serial passages. (B) The elevated endogenous ALDH1 ${ }^{+}$cells in let-7d/SPONGE were assessed by flow cytometry. (C and D) Control and let-7d-SPONGE OSCC cells were seeded in a 96-well plate and subjected to (C) cisplatin and (D) 5-FU treatment with different concentrations for $24 \mathrm{~h}$. The survival rate was determined by MTT assay $\left({ }^{*} \mathrm{P}<0.05\right)$. 
A

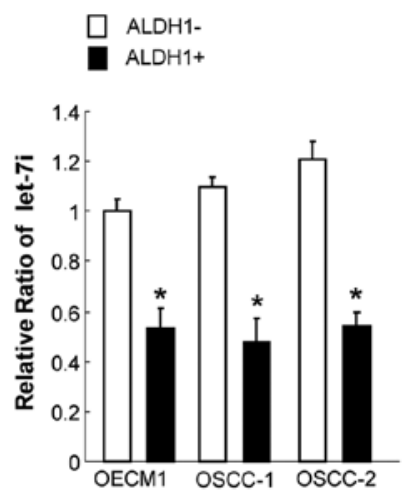

B

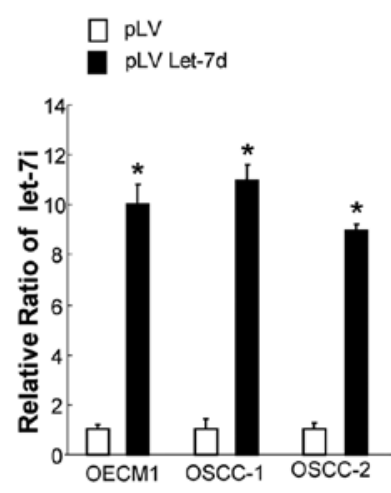

C

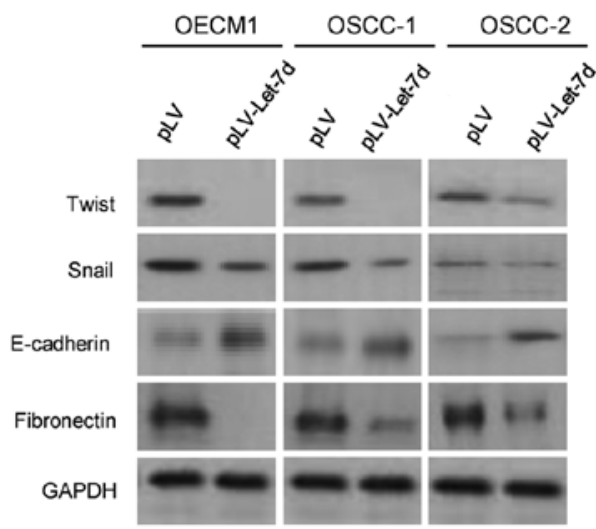

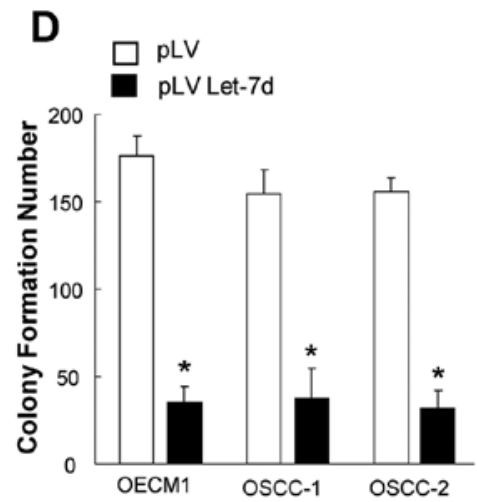
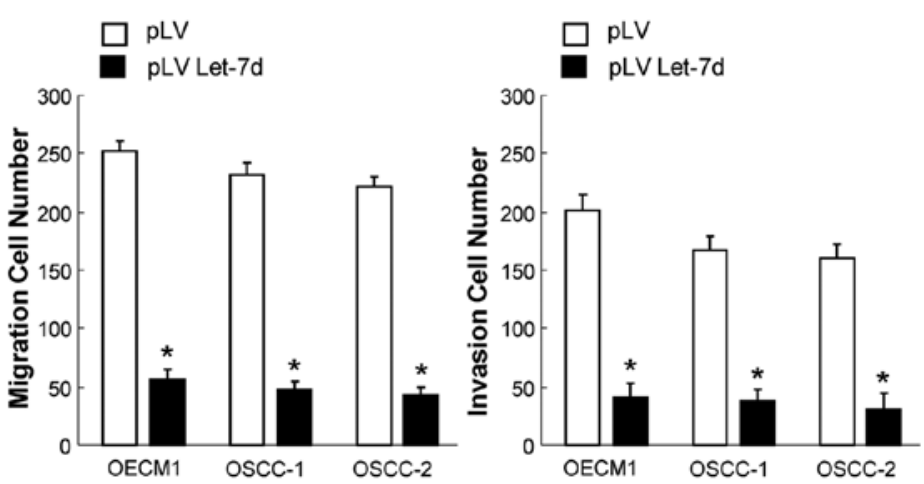

Figure 4. Down-regulation of self-renewal properties and enhancement of chemosensitivity of ALDH1 ${ }^{+}$OSCCs by let-7d. (A) Let-7d miRNA expression in control and let-7d-overexpressing OSCC-derived $\mathrm{ALDH}^{+}$cells by real-time RT-PCR. (B) Lentivirus-mediated transfection of OSSC-derived ALDHI ${ }^{+}$cells overexpress let-7d. (C) Expression EMT markers (Snail, Twist, N-cadherin and Fibronectin) of control and let-7d-overexpressing ALDH1 ${ }^{+}$cells were assessed by Western blotting. (D) Single cell suspension of ALDH1 ${ }^{+}$and ALDH1 $1^{+} /$let-7d cells were subjected to colony formation (left panel), migration assay (middle panel) and Transwell invasion (right panel).

Silencing let-7d enhances mesenchymal, stem-like, and chemo-resistant traits in OSCCs. To further investigate the correlations of Twist and Snail with let-7d expression level, we applied miRNA SPONGE strategy to specifically knock down endogenous let-7d in OECM1 and two primary cultivated OSCC cells. The efficiency of SPONGE-mediated let-7d knockdown was verified (Fig. 2A). The Western blotting of the three post-SPONGE treated clones all showed significant elevated of Twist and Snail levels (Fig. 2B). Epithelial-mesenchymal cellular transformation was also found increased after SPONGE-let-7d treatment of OSCC cells (Fig. 2C). Silencing of let-7d in OSCC cells significantly increased the soft agar colony number, signifying reduced anchorage-dependence, and enhanced the invasive ability (Fig. 2D and E). Recent reports suggest that OSCC possess the potential of cancer stem cell (CSC) or the tumor initiating cell-like properties, and presents characteristics of aggressive tumor $(22,26)$. Based on these studies as well as our finding that let-7d level is low in metastatic OSCC patients, we further explored the relationship between let-7d and cancer stemness properties of OSCC. The sphere formation ability of let-7d knockdown OSCCs in suspended culture was increased and lasted for several passages of culture (Fig. 3A), indicating enhanced self-renewal capacity. Moreover, silencing of let-7d in OSCC cells significantly increased the population of ALDH1-positive cells (Fig. 3B). To prove that knockdown of let-7d increases the stemness properties, we further elucidated the mechanisms how the chemoresistance was regulated by let-7d expression, as discovered by other researchers $(28,29)$. The CSC hypothesis has been bolstered by the clinical observation that malignant tumors are relatively resistant to chemotherapies, which has then become one of the functional definitions of CSC (30). Let-7d knockdown in OSCC increased its chemo-resistance to cisplatin and 5-FU (Fig. 3C and D). Collectively, these data implied that the silencing of let-7d renders OSCC capable of acquiring EMT and chemoresistant properties, and that this regulation could be modulated by elevated Twist and Snail and self-renewal properties.

Let-7d represses EMT and invasive ability in oral cancer tumor initiating cells. Based on the previous experimental results, we speculated that let-7d might regulate OSCC progression by regulating EMT, cancer stem-like properties, and chemoresistant in OSCC. To further address this issue, we initially isolated $\mathrm{ALDH}^{+}$cells using flow cytometry and cell sorting from primary OSCC cells as previously described (23). As expected, let-7d expression was dramatically reduced in $\mathrm{ALDH}^{+}$cells, whereas ALDH1 ${ }^{-}$cells showed higher let-7d expression (Fig. 4A). To elucidate whether let-7d was a strong mediator for OSCC-ALDH1 ${ }^{+}$cells, lentivirus-mediated 
A

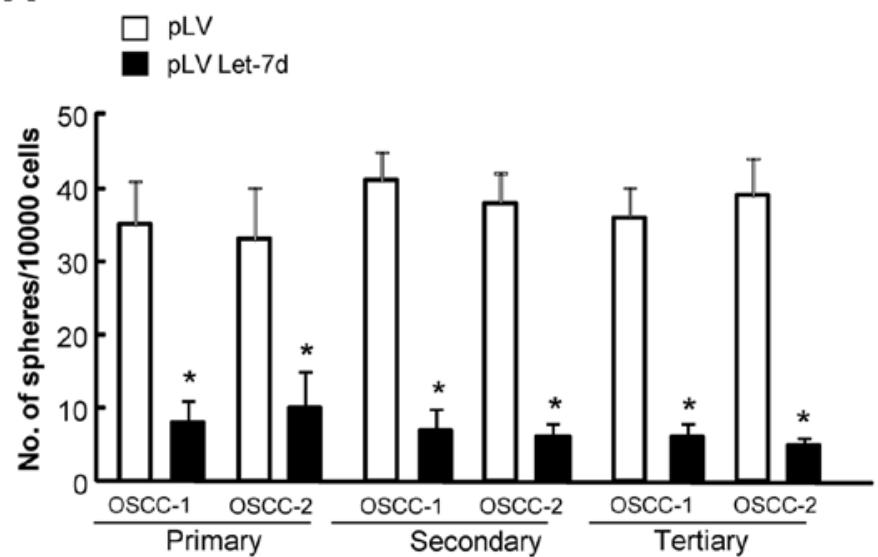

B

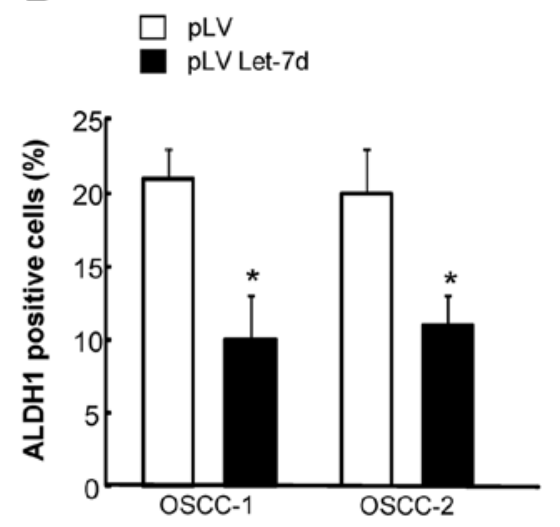

C

D
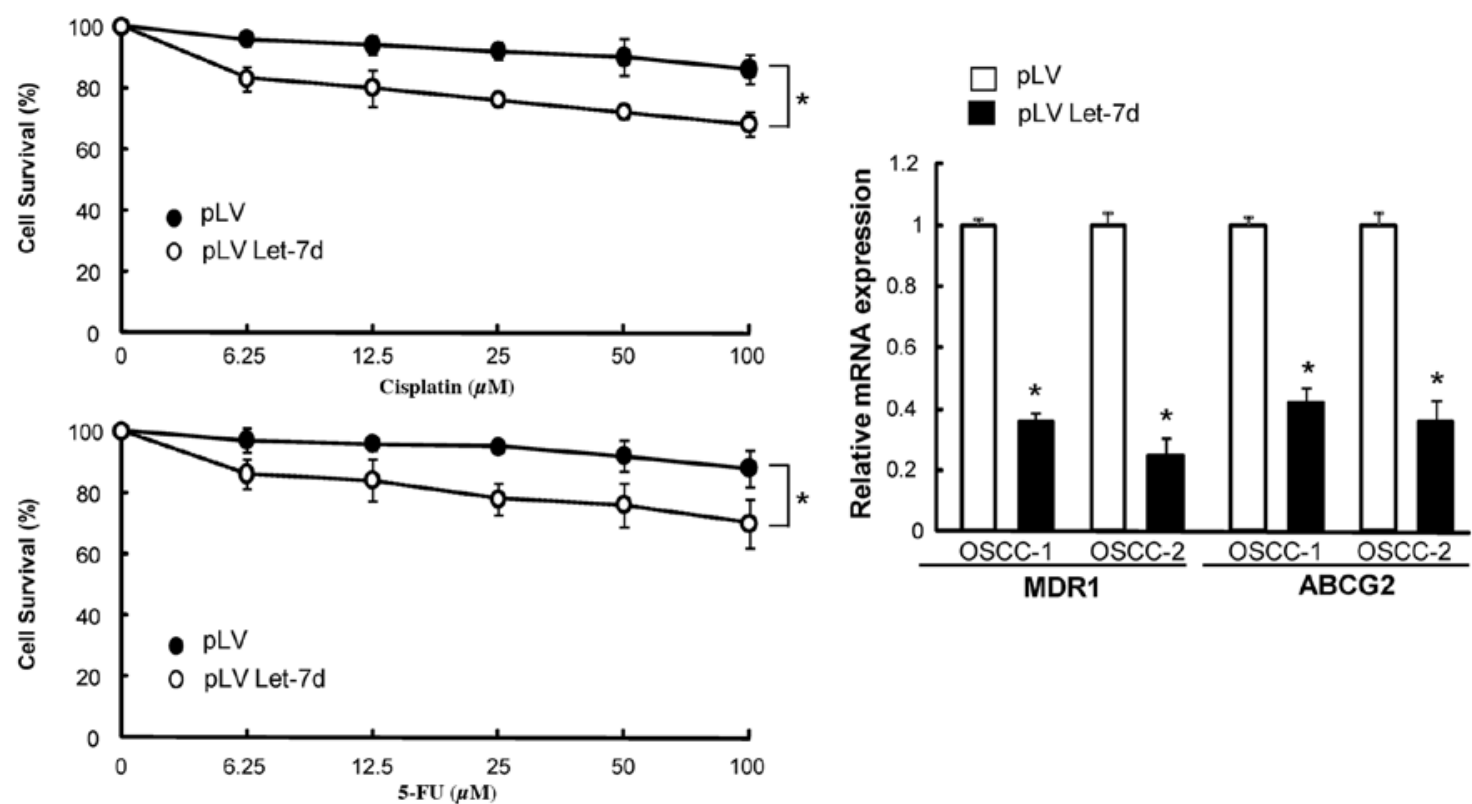

Figure 5. Modulation of the self-renewal and chemoresistant ability of ALDH1 ${ }^{+}$cells by let-7d. (A) Evaluation of the abilities for sphere body after serial

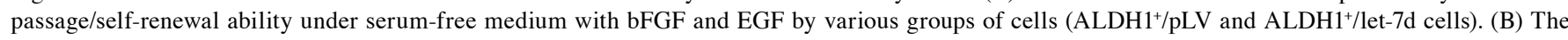
expressions of ALDH1 was validated in let-7d-overexpressing OSCC cells. (C) Both 10,000 control ALDH1 ${ }^{+}$and let-7d-overexpressing ALDH1 ${ }^{+}$cells were cultivated in a 96-well plate and treated with various concentrations of cisplatin (upper) and 5-FU (lower) for $24 \mathrm{~h}$ in 10\% FBS/DMEM/F-12 medium. The survival rate was determined by MTT assay. (D) The expression of MDR1 and ABCG2 transcripts was detected by real-time RT-PCR analysis in OSCC$\mathrm{ALDH} 1^{+}$cells with various treatment as indicated.

transfection strategy was adopted to overexpress let-7d in OSCC-ALDH1 ${ }^{+}$cells (Fig. 4B). The overexpressed let-7d decreased its targets, Twist, Snail, Vimentin and N-cadherin (Fig. 4C). The colony formation, cell migration, and tumor sphere formation abilities were all decreased upon let-7d-overexpression (Fig. 4D).

Let-7d regulates self-renewal and chemosensitivity in oral cancer tumor initiating cells. Overexpression of let-7d in primary OSCC cells derived from two patients repressed the sphere formation ability over several passages of suspension culture as shown in Fig. 5A. The inhibited CSC properties could be a result of diminished CSC population, as we found that the percentage of $\mathrm{ALDH}^{+}$cells was reduced in let-7d-overexpressed OSCC cells (Fig. 5B). Cell viability experiments showed that ectopic overexpression of let-7d inhibited the chemo-resistance to cisplatin and paclitaxel in $\mathrm{OSCC}_{-} \mathrm{ALDH} 1^{+}$cells (Fig. 5C). The decreased chemo resistance could be a result of down-regulated multidrug resistance (MDR) genes (Fig. 5D).

\section{Discussion}

miRNAs, a class of highly conserved small RNA molecules, act as either oncogenes or tumour suppressors, rendering them prospective targets for therapeutic development in cancer (31). It has been found that expression of oncogenic miR-21 and miR-184 promotes tumorigenicity in OSCC $(32,33)$. Ectopic expression of tumour-suppressive miR-98, miR-137 and miR-193a resulted in a loss of cell growth of OSCC. Recent clinicopathological findings demonstrated that the down-regulation of miR-133a, miR-133b, miR-205 
and let-7d in OSCC tumors could be a signature utilized for predicting the prognosis of OSCC patients $(21,34)$. Therefore, it is of interest to ask whether there is microRNA involved in regulating chemo-resistance or metastatic properties, such as EMT, in OSCC tumor cells. The ubiquitously expressed let-7/ miR-98 miRNA was one of the first mammalian miRNAs to be identified (5-8). Several studies of various human cancers, including lung cancer, gastric cancer, ovarian cancer, colon cancer, leiomyoma and melanoma, have found that let-7 family members are down-regulated during cancer progression (5-13). In OSCC, dysregulation of let-7 influences patient survival $(35,36)$. Let-7 has also been shown to act as a tumor suppressor, most likely through targeting a number of genes with oncogenic activity such as RAS (5-8) or high mobility group (HMG) A2 (9,37-39). Christensen et al found that the polymorphism of the let-7 biding site (KRAS-LCS6) is associated with reduced survival in oral cancer patients (40). The research group of the University of Florida also discovered that the Dicer, an RNase III endonuclease required for miRNA maturation, is overexpressed in oral cancers due to downregulation of let-7b (41). However, the involvement of let-7d in OSCC is still not clear. In this study, we first demonstrated that the expression of let-7d in the metastatic tumors of OSCC tissues was significantly decreased while the expressions of Twist and Snail were increased, as compared to the primary tumors (Fig. 1). EMT has also been considered as a key mechanism responsible for the process of cancer metastasis (42). Enhanced EMT characteristics is associated with poor overall and metastasis-free survival in patients with NSCLC (43). The role of stemness signature in EMT process has recently gained huge interest in the field of cancer research, as it also contributes to tumor metastasis and recurrence, making cancer difficult to tackle. It has been suggested that EMT can promote stemness property in normal breast tissues as well as breast cancer cells (44). Detailed bioinformatics analysis done by Ben-Porath et al discovered an embryonic stem cell-like gene expression signature in poorly differentiated aggressive tumors, including breast cancer, bladder cancer, breast cancer and brain tumors (45). Twist is a basic-helix-loop-helix (bHLH) transcription factor associated with cell lineage determination and differentiation. The protein encoded by this gene is a bHLH transcription factor and shares similarity with another bHLH transcription factor, Dermol. The strongest expression of this mRNA is in placental tissue; in adults, mesodermally derived tissues express this mRNA preferentially. Yang et al found Twist1 and Bmil was mutually essential to promote EMT and tumor-initiating capability (46). Bmil is a member of the Polycomb (PcG) family of transcriptional repressors that mediate gene silencing by regulating chromatin structure, and is essential for maintaining the self-renewal capacity of neural, hematopoietic, and intestinal stem cells. Bmil was originally identified as a proto-oncogene that cooperates with Myc to promote the tumorigenesis of lymphomas (47). Our experiments of SPONGE-mediated let-7d silencing further confirmed the reverse correlation between let-7d and Twist and Snail (Figs. 2 and 4). Further research is needed to identify the correlation of Bmil and let-7d in OSCC. Meanwhile, the phenotypic change of the OSCC cells from the epithelial-like to mesenchymal-like cell type were also found after let-7d-SPONGE applications (Fig. 2C). Because Twist and Snail are well-known genes that regulate the process of EMT, we then further elucidated the roll of Bmil and let-7d in EMT.

Furthermore, the chemo resistance property was modulated by let-7d in OSCC cell or OSCC-derived TICs (Figs. 3 and 5). The correlation between chemoresistance and the expression of specific miRNAs have been previously found by many researchers. Zhou et al discovered that suppression of proapoptotic Bcl-2 antagonist killer 1 (Bak1) expression which was induced by overexpressing miR-125b increases the chemoresistance of breast cancer cells to Paclitaxel (48), miR-21 $(49,50)$, miR-181a (51), miR-192 (52), miR-212 (53), miR-215 (52), miR-221/222 (54), miR-296 (55), miR-374a (51), miR-519a (51) and miR-630 (51) were proved to regulate the chemoresistances of pancreas, colorectal, head and neck, breast, and esophageal cancers to the treatments of gemcitabine, 5-fluorouracil, cisplatin, cetuximab, fulvestrant, and P-glycoprotein-related and -nonrelated drugs, respectively. Collectively, these results indicate that let-7d negatively modulates drug-resistant gene expression, playing a role in regulating the chemo-resistant ability in OSCC.

It is important to further elucidate the mechanisms by which downstream factors are regulated by let-7d in OSCC in vivo, and to investigate how let-7d modulates the chemoresistance, EMT, and self-renewal capacities in OSCC. Let-7d in the malignant OSCC or recurrent OSCC after chemotherapy may be useful in the future as a potential anti-cancer therapeutic strategy. Overall, restoration of let-7d in malignant oral cancer may initiate a new approach for therapeutic treatment in the future.

\section{Acknowledgements}

This study was supported by research grants from Tri-Service General Hospital (98/99/100), Chi Mei Medical Center (CMYM 9801/9901).

\section{References}

1. Jemal A, Siegel R, Ward E, et al: Cancer statistics, 2008. CA Cancer J Clin 58: 71-96, 2008.

2. Perez-Sayans M, Somoza-Martin JM, Barros-Angueira F, Reboiras-Lopez MD, Gandara Rey JM and Garcia-Garcia A: Genetic and molecular alterations associated with oral squamous cell cancer (Review). Oncol Rep 22: 1277-1282, 2009.

3. Visvader JE and Lindeman GJ: Cancer stem cells in solid tumours: accumulating evidence and unresolved questions. Nat Rev Cancer 8: 755-768, 2008.

4. Croce CM: Causes and consequences of microRNA dysregulation in cancer. Nat Rev Genet 10: 704-714, 2009.

5. Takamizawa J, Konishi H, Yanagisawa K, et al: Reduced expression of the let-7 microRNAs in human lung cancers in association with shortened postoperative survival. Cancer Res 64: 3753-3756, 2004.

6. Johnson SM, Grosshans H, Shingara J, et al: RAS is regulated by the let-7 microRNA family. Cell 120: 635-647, 2005.

7. Yanaihara N, Caplen N, Bowman E, et al: Unique microRNA molecular profiles in lung cancer diagnosis and prognosis. Cancer Cell 9: 189-198, 2006.

8. Akao Y, Nakagawa Y and Naoe T: Let-7 microRNA functions as a potential growth suppressor in human colon cancer cells. Biol Pharm Bull 29: 903-906, 2006.

9. Shell S, Park SM, Radjabi AR, et al: Let-7 expression defines two differentiation stages of cancer. Proc Natl Acad Sci USA 104: 11400-11405, 2007.

10. Motoyama K, Inoue H, Nakamura Y, Uetake H, Sugihara K and Mori M: Clinical significance of high mobility group A2 in human gastric cancer and its relationship to let-7 microRNA family. Clin Cancer Res 14: 2334-2340, 2008. 
11. Peng Y, Laser J, Shi G, et al: Antiproliferative effects by let-7 repression of high-mobility group A2 in uterine leiomyoma. Mol Cancer Res 6: 663-673, 2008.

12. Schultz J, Lorenz P, Gross G, Ibrahim S and Kunz M: MicroRNA let-7b targets important cell cycle molecules in malignant melanoma cells and interferes with anchorage-independent growth. Cell Res 18: 549-557, 2008.

13. Nam EJ, Yoon H, Kim SW, et al: MicroRNA expression profiles in serous ovarian carcinoma. Clin Cancer Res 14: 2690-2695, 2008.

14. Yu ML, Wang JF, Wang GK, et al: Vascular smooth muscle cell proliferation is influenced by let-7d microRNA and its interaction with KRAS. Circ J 75: 703-709, 2011.

15. Pandit KV, Corcoran D, Yousef $\mathrm{H}$, et al: Inhibition and role of let-7d in idiopathic pulmonary fibrosis. Am J Respir Crit Care Med 182: 220-229, 2010.

16. Lee S, Jung JW, Park SB, et al: Histone deacetylase regulates high mobility group A2-targeting microRNAs in human cord blood-derived multipotent stem cell aging. Cell Mol Life Sci 68 325-336, 2011

17. Li Y, VandenBoom TG II, Kong D, et al: Up-regulation of miR-200 and let-7 by natural agents leads to the reversal of epithelial-to-mesenchymal transition in gemcitabine-resistant pancreatic cancer cells. Cancer Res 69: 6704-6712, 2009.

18. Shao M, Rossi S, Chelladurai B, et al: PDGF induced microRNA alterations in cancer cells. Nucleic Acids Res: Jan 25, 2011 (Epub ahead of print)

19. Davidson LA, Wang N, Shah MS, Lupton JR, Ivanov I and Chapkin RS: n-3 Polyunsaturated fatty acids modulate carcinogen-directed non-coding microRNA signatures in rat colon. Carcinogenesis 30: 2077-2084, 2009.

20. Garzon R, Pichiorri F, Palumbo T, et al: MicroRNA gene expression during retinoic acid-induced differentiation of human acute promyelocytic leukemia. Oncogene 26: 4148-4157, 2007.

21. Childs G, Fazzari M, Kung G, et al: Low-level expression of microRNAs let-7d and miR-205 are prognostic markers of head and neck squamous cell carcinoma. Am J Pathol 174: 736-745, 2009.

22. Lo WL, Yu CC, Chiou GY, et al: MicroRNA-200c attenuates tumour growth and metastasis of presumptive head and neck squamous cell carcinoma stem cells. J Pathol 223: 482-495, 2011.

23. Yu CC, Lo WL, Chen YW, et al: Bmi-1 regulates snail expression and promotes metastasis ability in head and neck squamous cancer-derived ALDH1 positive cells. J Oncol 2011: 609259, 2011

24. Ebert MS, Neilson JR and Sharp PA: MicroRNA sponges: competitive inhibitors of small RNAs in mammalian cells. Nat Methods 4: 721-726, 2007.

25. Tsai LL, Yu CC, Chang YC, Yu CH and Chou MY: Markedly increased Oct4 and Nanog expression correlates with cisplatin resistance in oral squamous cell carcinoma. J Oral Pathol Med: Feb 22, 2011 (Epub ahead of print).

26. Wu MJ, Jan CI, Tsay YG, et al: Elimination of head and neck cancer initiating cells through targeting glucose regulated protein78 signaling. Mol Cancer 9: 283, 2010.

27. Polyak K and Weinberg RA: Transitions between epithelial and mesenchymal states: acquisition of malignant and stem cell traits. Nat Rev Cancer 9: 265-273, 2009.

28. Blower PE, Chung JH, Verducci JS, et al: MicroRNAs modulate the chemosensitivity of tumor cells. Mol Cancer Ther 7: 1-9, 2008.

29. Wendler A, Keller D, Albrecht C, Peluso JJ and Wehling M: Involvement of let-7/miR-98 microRNAs in the regulation of progesterone receptor membrane component 1 expression in ovarian cancer cells. Oncol Rep 25: 273-279, 2011.

30. Feng D, Peng C, Li C, et al: Identification and characterization of cancer stem-like cells from primary carcinoma of the cervix uteri. Oncol Rep 22: 1129-1134, 2009

31. Chen LH, Tsai KL, Chen YW, et al: MicroRNA as a novel modulator in head and neck squamous carcinoma. J Oncol 2010 $135632,2010$.

32. Li J, Huang H, Sun L, et al: miR-21 indicates poor prognosis in tongue squamous cell carcinomas as an apoptosis inhibitor. Clin Cancer Res 15: 3998-4008, 2009.

33. Wong TS, Liu XB, Wong BY, Ng RW, Yuen AP and Wei WI: Mature miR-184 as potential oncogenic microRNA of squamous cell carcinoma of tongue. Clin Cancer Res 14: 2588-2592, 2008.
34. Wong TS, Liu XB, Chung-Wai Ho A, Po-Wing Yuen A, Wai-Man Ng R and Ignace Wei W: Identification of pyruvate kinase type M2 as potential oncoprotein in squamous cell carcinoma of tongue through microRNA profiling. Int J Cancer 123: 251-257, 2008.

35. Wald AI,Hoskins EE, Wells SI, Ferris RL and Khan SA: Alteration of microRNA profiles in squamous cell carcinoma of the head and neck cell lines by human papillomavirus. Head Neck 33: 504-512, 2011.

36. Tran N, O'Brien CJ, Clark J and Rose B: Potential role of microRNAs in head and neck tumorigenesis. Head Neck 32: 1099-1111, 2010.

37. Kumar MS, Lu J, Mercer KL, Golub TR and Jacks T: Impaired microRNA processing enhances cellular transformation and tumorigenesis. Nat Genet 39: 673-677, 2007.

38. Mayr C, Hemann MT and Bartel DP: Disrupting the pairing between let-7 and Hmga 2 enhances oncogenic transformation. Science 315: 1576-1579, 2007.

39. Lee YS and Dutta A: The tumor suppressor microRNA let-7 represses the HMGA2 oncogene. Genes Dev 21: 1025-1030, 2007.

40. Christensen BC, Moyer BJ, Avissar M, et al: A let-7 microRNAbinding site polymorphism in the KRAS 3' UTR is associated with reduced survival in oral cancers. Carcinogenesis 30: 1003-1007, 2009.

41. Jakymiw A, Patel RS, Deming N, et al: Overexpression of dicer as a result of reduced let-7 MicroRNA levels contributes to increased cell proliferation of oral cancer cells. Genes Chromosomes Cancer 49: 549-559, 2010.

42. Thiery JP: Epithelial-mesenchymal transitions in tumour progression. Nat Rev Cancer 2: 442-454, 2002.

43. Wang SP, Wang WL, Chang YL, et al: p53 controls cancer cell invasion by inducing the MDM2-mediated degradation of Slug. Nat Cell Biol 11: 694-704, 2009.

44. Mani SA, Guo W, Liao MJ, et al: The epithelial-mesenchymal transition generates cells with properties of stem cells. Cell 133: 704-715, 2008

45. Ben-Porath I, Thomson MW, Carey VJ, et al: An embryonic stem cell-like gene expression signature in poorly differentiated aggressive human tumors. Nat Genet 40: 499-507, 2008

46. Yang MH, Hsu DS, Wang HW, et al: Bmil is essential in Twist1induced epithelial-mesenchymal transition. Nat Cell Biol 12: 982-992, 2010

47. Molofsky AV, Pardal R, Iwashita T, Park IK, Clarke MF and Morrison SJ: Bmi-1 dependence distinguishes neural stem cell self-renewal from progenitor proliferation. Nature 425: 962-967, 2003.

48. Zhou M, Liu Z, Zhao Y, et al: MicroRNA-125b confers the resistance of breast cancer cells to paclitaxel through suppression of pro-apoptotic Bcl-2 antagonist killer 1 (Bak1) expression. J Biol Chem 285: 21496-21507, 2010

49. Giovannetti E, Funel N, Peters GJ, et al: MicroRNA-21 in pancreatic cancer: correlation with clinical outcome and pharmacologic aspects underlying its role in the modulation of gemcitabine activity. Cancer Res 70: 4528-4538, 2010

50. Valeri N, Gasparini P, Braconi C, et al: MicroRNA-21 induces resistance to 5 -fluorouracil by down-regulating human DNA MutS homolog 2 (hMSH2). Proc Natl Acad Sci USA 107: 21098-21103, 2010.

51. Huang Y, Chuang A, Hao H, et al: Phospho- $\Delta \mathrm{Np} 63 \alpha$ is a key regulator of the cisplatin-induced microRNAome in cancer cells Cell Death Differ: Jan 28, 2011 (Epub ahead of print).

52. Boni V, Bitarte N, Cristobal I, et al: $\mathrm{miR}-192 / \mathrm{miR}-215$ influence 5-fluorouracil resistance through cell cycle-mediated mechanisms complementary to its post-transcriptional thymidilate synthase regulation. Mol Cancer Ther 9: 2265-2275, 2010.

53. Hatakeyama $\mathrm{H}$, Cheng $\mathrm{H}$, Wirth $\mathrm{P}$, et al: Regulation of heparinbinding EGF-like growth factor by miR-212 and acquired cetuximab-resistance in head and neck squamous cell carcinoma. PLoS One 5: e12702, 2010.

54. Rao X, Di Leva G, Li M, et al: MicroRNA-221/222 confers breast cancer fulvestrant resistance by regulating multiple signaling pathways. Oncogene 30: 1082-1097, 2011.

55. Hong L, Han Y, Zhang H, et al: The prognostic and chemotherapeutic value of miR-296 in esophageal squamous cell carcinoma. Ann Surg 251: 1056-1063, 2010. 